\title{
Now, Factuality and Conditio Humana
}

\author{
Hartmann Römer \\ Institute of Physics, University of Freiburg, Germany
}

\begin{abstract}
The relationship between inner and outer time is discussed. Inner time is intrinsically future directed and possesses the quality of a distinguished "now". Both of these qualities get lost in the operationalized external physical time, which, advancing towards more fundamental physics, tends to become more similar to space and even fade away as a fundamental notion. However, inner time as a constitutive feature of human existence holds its place in the heart of quantum theory and thermodynamics.
\end{abstract}

\section{Introduction}

Time lies next to the hot focus of our mode of existence as conscious beings and has always been a permanent subject of human thinking and philosophy. Our world and even our own mind is given to us in the inexorably temporal form of a movie-like course of appearances rather than as a simultaneous panoramic picture. All our reflections about this temporality are caught inside the genuinely temporal structure of a stream of consciousness. In fact, trying to escape temporality by an effort of thought is an extremely delicate task at the verge of paradox and unthinkability.

Over the centuries, much philosophical activity was devoted to a detailed analysis of the temporal mode of human existence. Prominent names as Augustine, Kant, Hegel, Bergson and Heidegger are witnesses of this endeavor. Employing the terminology of Mc Taggart[1], internal existential time of man is an A-time, characterized by future directedness and in particular by the existence of the temporal quality of a distinguished "now". The window of this "now" moves forward into the future leaving past behind it. The feeling of this flow is what remains, if all sensory input is neutralized. According to Heidegger the driving force behind this motion of the "now" is not a push from the past but rather a pull from the future originating in the fundamental structural feature 
of "worry"(German: "Sorge") of human existence.

One particularly important fruit of the persistent philosophical concern about time is the emergence, clarification and sharpening of the concept of outer or physical time. Here time has been tamed, harnessed and subdued to measurability by clocks. External physical time differs from internal time in several respects. Again using Mc Taggart's terminology, it can be denoted as a B-time, a scale time representable by the set of points on a line or a set of real numbers. There is no quality of a distinguished "now", all time points are equivalent just as points on the line. Moreover, future directedness is not necessarily related to physical B-time. The concept of physical time has been spectacularly successful. Its power manifests itself in the omnipresence of clocks which hold all of us under their sway. There is a common tendency to accept physical B-time as the only exact and in a sense only real notion of time and to reduce internal A-time to a subordinate or even illusionary status. Investigating the relationship between internal and physical time will be the main subject of this note.

\section{2 "Spacialization" and evaporation of physical time}

Along with the advancement towards more fundamental levels of physics, physical B-time shows a tendency to become more and more similar to space. In addition, there are strong indications, that, together with space, it might disappear altogether as a fundamental notion, when pushed to the extremes of cosmology and quantum gravity.

From the outset, the lack of a privileged "now" in B-time parallels the absence of a privileged "here" in space. In this respect, B-time is more similar to space than A-time. This opens up the possibility to represent processes in time in a diagrammatic atemporal way by introducing a spacial time axis.

Let us first look at the motion of a point particle. The presence of this particle in a point $\mathrm{x}$ at (or very close to) time $\mathrm{t}$ is an example of a point event with a precise localization in time and space, which can be described by four coordinates, one temporal coordinate and three spacial coordinates. Then the motion of the point particle is completely described by its world line, a one-dimensional set of point events giving the position of the particle at every time (see figure 1). This world line, as it stands, is a line in space, for instance on a sheet of paper and as such entirely timeless. Reference to time is only given by its interpretation as a representation of a motion.

This possibility of a timeless representation of processes is not restricted to physical

motions. For instance, the phylogeny of man can be adequately represented by an totally timeless family tree (see Figure 2). There is no evident way to decide, which representation 


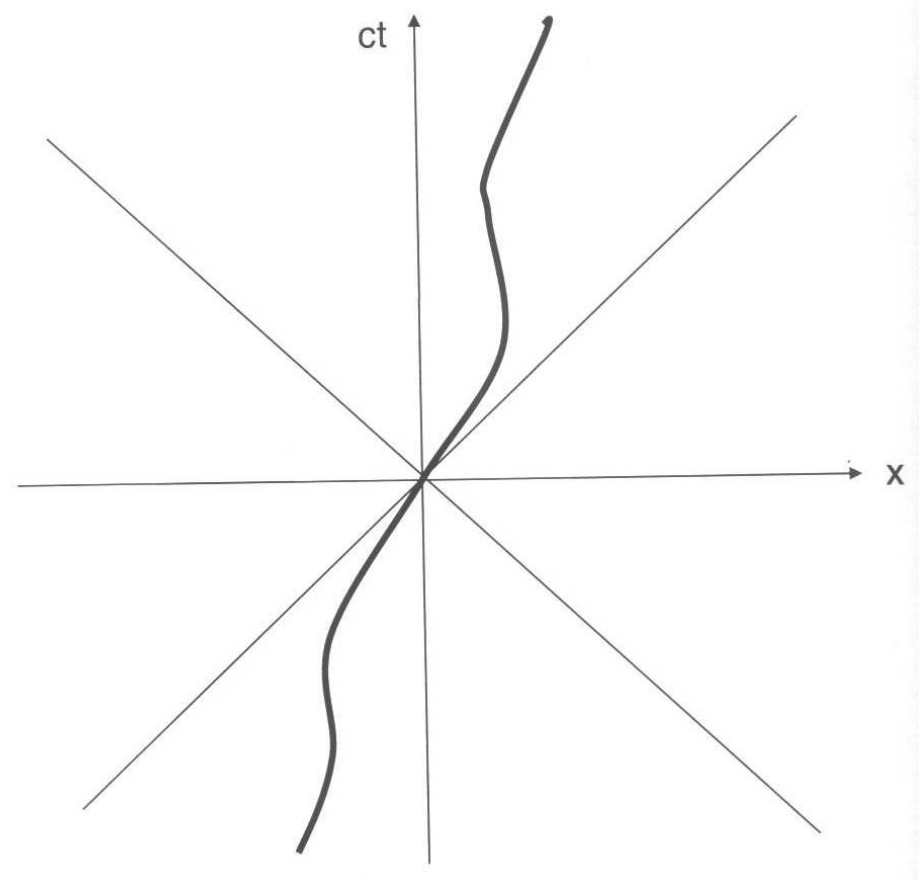

Figure 1: Motion of a point particle in the timeless world line representation. Two space dimensions are suppressed. 


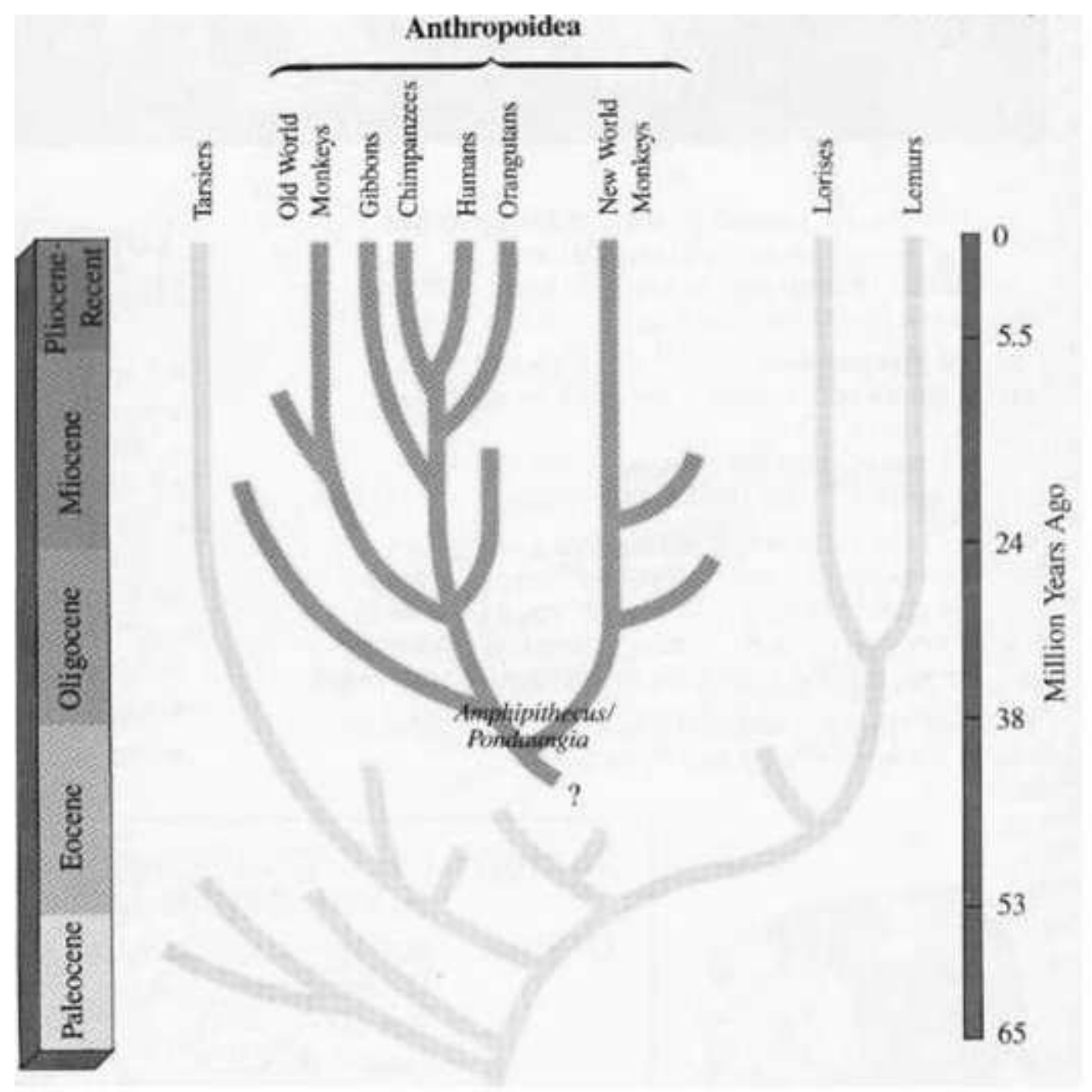

Figure 2: Development of hominides as a timeless family tree 
is more correct or "real".

Passing from Newtonean space-time to space-time in Special and General Relativity, one observes that time becomes more and more similar to space.

From the perspective of Kant's philosophy this is not a complete surprise: According to Kant time and space are forms of intuition (German: "Anschauungsformen"), through which everything has to pass, which reaches our mind. Time is the form of the inner sense and space the form of the outer sense. (The Kantian distinction between inner and outer sense is akin to the Cartesian distinction between res cogitans and res extensa.) As physics is concerned with the outer world, externalized physical time can be expected to become more similar to space.

Let us now follow the increasing "spacialization" of time from Newtonean mechanics to General Relativity Theory in more detail.

a) In Newtonean space time the transition between the coordinates of point events in different inertial systems is performed by Galilei-transformations. It is possible to define a global Newtonean world time attributing a time coordinate to every point event such that time differences $\tau_{i, j}=t_{i}-t_{j}$ between any two point events are independent of the inertial system. This implies in particular, that the simultaneity $\tau_{i, j}=0$ of two events is an invariant notion. Moreover, one immediately obtains

$$
\tau_{1,2}+\tau_{2,3}=\tau_{1,3}
$$

Space behaves differently under Galilei-transformations. Spacial coincidence is a relative, system dependent notion: Point events occurring at the same space point in one inertial frame will in general occur at different space points in another inertial frame. Only for simultaneous events the spacial distance $r_{i, j}=\left|\vec{x}_{i}-\vec{x}_{j}\right|$ has an invariant meaning. Instead of equation (11) one has the triangular inequality

$$
r_{1,2}+r_{2,3} \geq r_{1,3}
$$

b) In Special Relativity Theory the transition between different inertial systems is performed by proper orthochronous Poincaré-transformations. Time differences are no longer invariant but depend on the inertial system just like spacial distances. In particular, simultaneity becomes a relative, system dependent notion. To be more concrete, the situation in Special Relativity Theory is as follows:

Light propagates with the same velocity $c$ in all inertial systems, and this velocity $c$ is the highest possible velocity for any signal. Two different point events $e_{i}$ and $e_{j}$ are called relative timelike, if a subluminar signal can be exchanged between them, relative lightlike, if only a luminal signal can be exchanged between them and relative spacelike, if they 
cannot be connected by a signal. It turns out, that the temporal order of relative timelike or lightlike signals is independent of the inertial systems. In other words, the sign (but not magnitude) of the time coordinate difference of such signals is invariant. This assures that the temporal order of cause and effect is the same in every inertial system as it should. On the other hand, the temporal order of relative spacelike events is system dependent. It is always possible to change the sign of time coordinate differences of such events by a change of the inertial system, and there is always an inertial system, in which relative spacelike events are simultaneous. ( Of course simultaneous events are relative spacelike, because evidently a signal connecting them would have to have infinite velocity.)

For relative timelike events $e_{i}$ and $e_{j}$ there is an invariant measure of their temporal distance: $\tau_{i, j}$ is defined as the so-called proper time measured along a geodesic, i.e. straight world line connecting $e_{i}$ and $e_{j}$. If $e_{2}$ lies in the future of $e_{1}$ and $e_{3}$ in the future of $e_{2}$, then rather than the equality (1) an inequality

$$
\tau_{1,2}+\tau_{2,3} \leq \tau_{1,3}
$$

holds, a triangular inequality similar to the spacial inequality (2) but with a $\leq$ instead of a $\geq \operatorname{sign}$. (This $\leq$ sign is the origin of the famous twin paradox: The proper time difference for a worldline connecting two relative timelike events is maximal for a geodesic.) For relative lightlike events $\tau_{i, j}=0$, and (3) remains valid, if we allow some of the proper time differences in it to vanish.

For relative spacelike events $e_{i}$ and $e_{j}$ it is possible to define an invariant spacial distance $r_{i, j}$, which is simply the spatial distance in an inertial system in which $e_{i}$ and $e_{j}$ are simultaneous. The spacial triangular inequality (2) remains valid as long as $e_{1}, e_{2}$ and $e_{3}$ lie in one spacelike plane or, equivalently, if all three events are simultaneous in an appropriate inertial system. So, we notice yet another similarity between time and space in Special Relativity Theory: Both time- and space- differences fulfill triangular inequalities.

c) The unification of space and time goes even further in General Relativity Theory. Global inertial coordinate systems are no longer definable, but an observer, freely falling in a gravitational field, can at least realize a local inertial system, in which the laws of Special Relativity are valid for small deviations from the origin of the space time coordinates. This is just a reformulation of Einstein's equivalence principle. The concept of signals and the distinction between relative timelike, lightlike and spacelike events remains valid. Space and time are fused in the geometric concept of a pseudoriemannian space-time manifold $M$ and point events are points in $M$. On $M$ a metric tensor $g_{\mu \nu}$ is defined, which allows to measure the length of world lines in $M$. Also the concepts of geodesics and geodesic distances remain well defined and the inequalities (2) and (3) still hold (at least if $e_{1}, e_{2}$ and $e_{3}$ are not too far apart). Timelike and lightlike geodesics are the worldlines of massive particles and photons respectively in a gravitational field. 
In sharp contrast to Newtonean physics and Special Relativity, space-time is no longer the external arena on which the play of physics is performed, but the arena takes part in the play: The curvature of the space-time metric, which measures its deviation from the "flat" geometry of Special Relativity depends on the distribution of energy and momentum of matter in the physical universe. The view of the universe as suggested by General Relativity is a timeless geometric structure often referred to as a block universe. The dynamics of particle motions appears to be frozen in a concept of world lines in the space-time manifold $M$, which does not suggest any natural definition of a time coordinate. Also fields like the electromagnetic field or the gravitational field appear in the timeless geometric mathematical form of sections in certain bundles over the space-time manifold $M$.

Physical B-time, as opposed to internal A-time is not necessarily directed. In fact, neither Newtonean time nor time in Special Relativity nor the concept of pseudoriemannian space-time manifolds in General Relativity is connected to any notion of a preferred time direction. Moreover, the fundamental laws of physics are invariant under time inversion. A tiny violation of time inversion invariance in weak interactions may look like a possible exception, but this asymmetry is probably due to spontaneous symmetry breaking: It results not from an asymmetry of the fundamental equations of physics but from an asymmetry of their solution. This also applies to the apparent asymmetry imposed by the space-time metric of the expanding universe, which is a time asymmetric solution of the symmetric Einstein equations of General Relativity. The directedness of time coming from the second law of thermodynamics will be discussed later.

One of the virtues of General Relativity Theory is that it is able to predict borders of its validity. The singularity theorems of Hawking and Penrose prove that certain world lines will inevitably terminate in space-time singularities, where curvature quantities become infinite. This, for instance, occurs, when black holes are formed. Also, all time- or lightlike worldlines of the visual parts of our universe originate in a gigantic "big bang" singularity. No time parameter can be extended before this singularity. Moreover, adding some elements of quantum theory, one sees that the "spacialization" of time turns into an abdication and successive loss of both time and space. In fact, time and space lose their meaning for distances of the order of Planck's time $t_{P}=\sqrt{\hbar G / c^{5}} \approx 5.4 \cdot 10^{-44} \mathrm{~s}$ or Planck's length $l_{P}=c t_{P}=\sqrt{\hbar G / c^{3}} \approx 1.6 \cdot 10^{-35} \mathrm{~m}$, where $G$ is the gravitational constant and $\hbar$ Planck's quantum of action. This can be seen in the following way: In order to resolve spacial distances $l$ by a measurement, according to Heisenberg's uncertainty principle one has to provide momentum of order $\hbar / l$ in a volume $l^{3}$. By Einstein's equations of General Relativity this gives rise to a change of space-time curvature characterized by a curvature radius $L$ with $1 / L^{2}=G p / l^{3} c^{3}=\hbar G / l^{4} c^{3}$. The measurement of $l$ certainly 
becomes ill-defined if the resulting curvature change $L$ is of the order of magnitude of $l$. Equating $l$ and $L$ gives $l=L=l_{p}=\sqrt{\hbar G / c^{3}}$.

As a consequence, the classical General Relativity Theory becomes unreliable in a neighborhood of order $l_{P}$ or $t_{P}$ of the singularities predicted by this theory, including the big bang singularity.

Although quantum field theory on fixed pseudoriemannian space-time manifolds is by now well developed, quantum theory of space-time itself is still in its infancy. The preceding argument shows that quantum effects become dominant at distances of the order of magnitude of Planck's length and time. Also the distinction between timelike, spacelike and lightlike separation becomes blurred by quantum indeterminacies. The problem of finding a Quantum Gravity Theory[2], i.e. a quantum theory of space-time is unsolved and perhaps the greatest challenge of fundamental theoretical physics. Describing, comparing and assessing the various current approaches towards this problem would at least require a book and be way beyond the scope of this note. We restrict ourself to some short remarks about three different approaches.

a) String Theory [3] starts out from quantizing the motion of a one-dimensional string like object vibrating in a background space-time manifold $B$. Planck's length is closely related to the tension of this string. In fact, the string has to be supersymmetric and carries spin degrees of freedom. There are good arguments that the quantization of the string leads to a massless particle of spin 2 to be identified with the quantum of the gravitational field. (However, the emergence of curved four-dimensional space-time is not quite clear.) In addition, the claim is, that String Theory describes all particles and interactions of physics. Later on, it turned out, that for the sake of consistency the quantum theory of the string should also contain higher dimensional vibrating objects called branes. The technical and, more seriously, conceptual difficulties of String Theory are formidable. So far, no precise formulation of its theoretical framework is available. It is not yet clear how to get rid of the undesirable background dependence of string theory. For consistency, the background manifold $B$ must be ten-dimensional, and six space dimensions must be "compactified" i. e. curled up at a small scale in order not to be directly visible. The precise form of this compactification is undetermined and seems to be highly arbitrary. (According to rough estimates there are more than $10^{500}$ different compactifications.) The particle content and the interactions of the resulting theory radically depend on the compactification, leading to a loss of predictive power as long as no principle for identifying preferred compactifications can be given. To make things worse, no known compactification reproduces the well established standard model of elementary particle theory.

b) Loop Quantum Gravity [4, 5] belongs to a class of theories which assume, that for very small scales of the order of magnitude of $l_{P}$ space-time or space loses its continuous man- 
ifold character and becomes discontinuous and discrete like a lattice. On larger scales the number of lattice points should become so large that in the average a continuum limit is a good approximation, such that a smooth manifold structure is regained. So, the smooth space time of Newtonean theory and Special and General Relativity is conceived as a derived, approximate notion. The virtue of such theories is that singularities at small scale are excluded from the outset. However, it is still not clear, how and under what conditions the smooth continuum limit is obtained.

c) Canonical Quantum Gravity tries to apply the procedure of canonical quantization to Einstein's theory of gravitation. In its most popular form, it leads to the Wheeler-de Witt equation [6], an equation of the structure

$$
H\left(g^{(3)}, \delta / \delta g^{(3)}\right) \Psi\left(g^{(3)}\right)=0 .
$$

Here, the "Schrödinger wave function" $\Psi\left(g^{(3)}\right)$ is a functional depending on metric $g^{(3)}$ on a three dimensional manifold, and the Hamiltonean $H\left(g^{(3)}, \delta / \delta g^{(3)}\right)$ depends on $g^{(3)}$ and the functional derivatives with respect to $g^{(3)}$. Unlike a normal Schrödinger equation in quantum mechanics, the Wheeler- de Witt equation contains no time derivative of the wave function and is, in this sense, completely timeless. This is related to the absence of any natural time coordinate in General Relativity. Something like time may be recuperated in a quasiclassical approximation (After all, the space-time we are living in is classical) of the type

$$
\Psi \sim \exp (i S / \hbar)
$$

In this case, the support of the action functional $S$ is decomposed into one-dimensional families of 3-metrics, which can be interpreted as metrics on 4-dimensional manifolds. Depending on the solution $\Psi$, the signature of the 4-metric may be pseudoeuclidean, and the parameter along such a one-dimensional family can be interpreted as a time coordinate. The Big Bang singularity may appear in the form of a boundary condition on $\Psi$. We see, that also in this approach, time only appears as a secondary, derived and approximate notion. In addition, there are also solutions of the Wheeler- de Witt equation which do not allow for the introduction of a time coordinate. It must also be admitted that the precise mathematical definition of the Hamiltonean and the wave functional in the Wheeler- de Witt equation is not yet clear.

These examples for Quantum Gravity theories clearly show, that the tendency of a successive abdication of physical time is continued, if quantum effects on space-time are taken into account. 


\section{The revival of A-time in quantum theory}

The progressive spacialization of physical time becomes also evident in a comparison of nonrelativistic quantum mechanics, quantum field theory on Minkowskian space and quantum field theory on curved background space-time manifolds.

Nonrelativistic quantum mechanics is the quantum version of Newtonean mechanics. Space and time play quite different roles: Whereas space is represented by position operators acting on the Hilbertspace of quantum states, time is present as a classical parameter $t$ of B-type. In the Schrödinger picture of quantum mechanics, the state vector $\psi(t)$ depends on this parameter. Alternatively, in the Heisenberg picture all observables depend on $t$.

In quantum field theory the Heisenberg picture is conceptually favored and both space and time are reduced to the same status of classical parameters such that the field operators $\Phi(t, \vec{x})$ are functions of $t$ and $\vec{x}$. It is still formally possible to construct spacial and temporal localization operators from these field operators [7, 8. but with a lot of arbitrariness. The similarity of the time and space parameters is further enhanced in special relativistic quantum field theory and even more so in quantum field theory on curved background manifolds. The parametric space time dependence is of paramount importance for the axiomatic formulation of local quantum field theory 9 . The concept of locality has to be revised, if also the geometry of time and space is subject to quantization and loses its fundamental meaning as we saw in the preceding paragraph. In view of the spacial character of physical (B-)time and its eventual disappearance, time is sometimes assumed to be unreal and illusionary. Attributing such an ontologically subordinate status to time signals a strong physicalistic and reductive attitude connected to the claim that, at least in principle, everything should be describable in terms of physics. We shall argue that this creed is of low plausibility. Right here we should notice that physics is a highly developed, sophisticated and spectacularly successful method to build up a mathematical model of the world. But by definition a model concentrates on features amenable to its framework, and it is certainly a methodological mistake to identify a model with what it modelizes.

Classical physics silently supposes an external observer merely registering phenomena of the observed system without influencing it in an essential and non-negligible way. This supposition is no longer tenable in quantum theory, where measurement will in general change even pure states. The role of the observer becomes an active rather than a merely registrating one, and time in the form of the A-time of the observer has its place right in the heart of quantum theory:

A measurement result is factual and appears via the "now" of the observer. The directedness of the observer's A-time is encoded in the non commutative structure of 
quantum theory. The order of successive measurements is vital, and in general only the result of the last and latest measurement can claim factual status, whereas the factuality of previous measurement results is destroyed by subsequent measurements. Notice, that the composition $P_{1} P_{2}$ of two proposition observables $P_{1}$ and $P_{2}$ means that $P_{1}$ is applied and measured after $P_{2}$.

A complete physical description of a measurement process in terms of quantum dynamics is not available. In quantum dynamics, time development is given by unitary transformations of states or observables. A measurement results in a non unitary reduction of the quantum state. Of course, a measurement process is accompanied by a quantum physical process, but this physical description does not seem to be exhaustive. In fact, no clear purely physical criterium lends itself to qualify a physical process as a measurement process. In addition to being a physical event, a measurement is also an act of cognition. In the subtle role of the measurement process, quantum physics has an open door to cognition and epistemology, and such philosophical considerations cannot be ignored as easily as in classical physics. Unitary time development of quantum dynamics is completely deterministic, and nondeterministic randomness enters only through measurement. Also the causal closure of pure quantum dynamics is broken by the measurement process and the freedom of a choice of the observable to be measured. (This also applies to classical theory but becomes more conspicuous in quantum theory.) The thermodynamic time arrow pointing towards an increase of entropy is another case, where an inroad of directed A-time into physics can be observed. The notion of entropy rests on an incomplete description of a system in terms of macroscopic states, where a complete description in terms of microscopic states is unfeasible. The transition from a microscopic to a macroscopic description is performed by the application of a coarse graining procedure, which associates a certain well-defined mixed statistical product state to the system and thereby a definite value to its entropy. Normal microscopic physical dynamics (unitary in quantum theory, symplectic in classical theory) does not change the entropy of this state. An increase of entropy only results when coarse graining is again applied to the time developed state. Thereby inaccessible correlation information between the different subsystems corresponding to the coarse graining is discarded and entropy is increased. So far, the reasoning was still time symmetric, because the microscopic physical time development could have been taken in both time directions. The directedness of A-time enters, because an observer can only first register a macroscopic state and then discard correlation information and not vice versa.

For an investigation of the relationship between inner A-time and outer physical B-time we need a comprehensive framework including both mental and material systems. Generalized Quantum Theory(GQT) [10, 11, 12] provides a general system theory, which arose from physical quantum theory in its algebraic form leaving out those features which 
only pertain to physical systems 1 The resulting formalism is still rich enough to allow a controlled and formally well defined application of quantum theoretical notions like complementarity and entanglement far beyond the realm of physics. Mental systems, in particular the human mind as seen from an inner first person perspective are well inside the scope of GQT and are even of paradigmatic importance in this framework.

\section{Generalized Quantum Theory}

In this section, we give a brief account of the vital structural features of GQT in order to ease the understanding of our argumentation in the subsequent sections. For a full account of GQT we refer to the original publications [10, 11, 12]. References for numerous applications of GQT, which have been worked out in more or less detail, can be found in [12 and [13. In [14] a new application of GQT to order effects in questionnaires is described. GQT takes over from quantum physics the following four fundamental notions:

1. System: A system is anything which can be (imagined to be) isolated from the rest of the world and be subject to an investigation. In the sequel we shall consider systems containing also conscious individuals. In contradistinction to, e.g., classical mechanics the identification of a system is not always a trivial procedure but sometimes a creative act. In many cases it is possible to define subsystems inside a system.

2. State: A system must have the capacity to reside in different states without losing its identity as a system. One may differentiate between pure states, which correspond to maximal possible knowledge of the system and mixed states corresponding to incomplete knowledge. In the most general form of GQT, the set of states has no underlying Hilbert state structure. For some applications (see, e.g., [15, 16, 17, 14]) one may want to enrich the minimal scheme of GQT, for instance by adding this additional structure.

3. Observable: An observable corresponds to a feature of a system, which can be investigated in a more or less meaningful way. Global observables pertain to the system as a whole, local observables pertain to subsystems.

\footnotetext{
${ }^{1}$ Originally it was called "Weak Quantum Theory", but this led to misunderstanding by non mathematicians. Admittedly, the term "Generalized Quantum Theory" is somewhat unspecific and equivocal and has been used in different senses by other authors.
} 
4. Measurement: Doing a measurement of an observable $A$ means performing the investigation which belongs to the observable $A$ and arriving at a result $a$, which can claim factual validity. What factual validity means, depends on the system: Validity of a measurement result for a system of physics, internal conviction for self observation, consensus for groups of human beings. The result of the measurement of $A$ will in general depend on the state $z$ of the system before the measurement but will not be completely determined by it. In GQT as well as in physical quantum theory, the notion of measurement contains an element of idealization, because measurement is not described as a temporal process, rather the focus of attention lies on the factual result obtained eventually.

In addition to these definitions the following structural features of GQT are of particular importance, generalizing essential properties of physical quantum theory. To every observable $A$ we associate its spectrum, a set $\operatorname{Spec} A$, which is just the set of all possible measurement results of $A$. Immediately after a measurement of an observable $A$ with result $a$ in $\operatorname{Spec} A$, the system will be in an eigenstate $z_{a}$ of the observable $A$ with eigenvalue $a$. The eigenstate $z_{a}$ is a state, for which an immediate repetition of the measurement of the same observable $A$ will again yield the same result $a$ with certainty, and after this repeated measurement the system will still be in the same state $z_{a}$. This property, which is also crucial in quantum physics justifies the terminology "eigenstate of an observable $A$ " for $z_{a}$ and "eigenvalue" for the result $a$. We repeat that this is an idealized description of a measurement process abstracting from its detailed temporal structure.

Two observables $A$ and $B$ are called complementary, if the corresponding measurements are not interchangeable. This means that the state of the system depends on the order in which the measurement results, say $a$ and $b$, were obtained. If the last measurement was a measurement of $A$, the system will end up in an eigenstate $z_{a}$ of $A$, and if the last measurement was a measurement of $B$, an eigenstate $z_{b}$ will result eventually. For complementary observables $A$ and $B$ there will be at least some eigenvalue, say $a$, of one of the observables for which no common eigenstate $z_{a b}$ of both observables exists. This means that it is not generally possible to ascribe sharp values to the complementary observables $A$ and $B$, although both of them may be equally important for the description of the system. This is the essence of quantum theoretical complementarity which is well defined also for GQT.

Non complementary observables, for which the order of measurement does not matter, are called compatible. After the measurement of compatible observables $A$ and $B$ with results $a$ and $b$, the system will be in the same common eigenstate $z_{a b}$ of $A$ and $B$ irrespective of the order in which the measurements were performed. 
In quantum physics, entanglement is normally explained by the existence of non separable Hilbert space states, which are linear superpositions of separable tensor product states. But entanglement can also be defined in the framework of GQT, which contains no reference to a Hilbert space of states [10, 11, 12, 18]. It may and will show up under the following conditions:

1. Subsystems can be identified within the system such that local observables $A_{i}$ pertaining to different subsystems are compatible.

2. There is a global observable $A$ of the total system, which is complementary to local observables $A_{i}$ of the different subsystems.

3. The system is in an entangled state, for instance in an eigenstate of the above mentioned global observable $A$, which is not an eigenstate of the local observables $A_{i}$.

Given these conditions, the measured values of the local observables will be uncertain because of the complementarity of the global and the local observables. However, socalled entanglement correlations will be observed between the measured values of the local observables $A_{i}$ pertaining to different subsystems. These correlations are non local and instantaneous.

In physical quantum theory, the singlett state of a two-spin system is a standard example of entanglement. In this case, the total spin

$$
S^{2}=\left(s_{1}^{(1)}+s_{1}^{(2)}\right)^{2}+\left(s_{2}^{(1)}+s_{2}^{(2)}\right)^{2}+\left(s_{3}^{(1)}+s_{3}^{(2)}\right)^{2}
$$

is the global observable, the individual spin observables $s_{3}^{(1)}$ and $s_{3}^{(2)}$ take over the role of the local observables complementary to the global observable, and the entangled singulett state is the eigenstate of $S^{2}$ with eigenvalue zero. We see how our generalized definition captures the essentials of quantum theoretical entanglement.

In physical quantum theory it is not difficult to show that entanglement correlations cannot be used for signal transmission or controlled causal influences. In order to avoid intervention paradoxes this must be postulated for GQT [19, 20, 18].

In view of the possibility of entanglement correlations in GQT the problematic and anything less than trivial character of the act of identification of a system becomes even more acute. 


\section{Observables, partitions and epistemic cut}

In classical or quantum mechanics, observables like positions and velocities seem to be given in a very direct and unproblematic way by the system itself. The example of entropy ${ }^{2}$ shows that even for physical systems the identification of observables need not be so trivial but sometimes means a major discovery. For very general systems like those considered in GQT, observables are not so directly given by the system and read off from it like location and velocity in a mechanical system. On the contrary, as already suggested by the name of an "observable", the identification of an observable may be a highly creative act of the observer, which will be essentially determined by his horizon of questions and expectations. This marks a decidedly epistemic trait of the notion of observables in GQT even more so than in quantum physics. Moreover, the horizon of the observer will change, not the least as a result of his previous observations adding to the open and dynamical character of the set of observables. In humanities, this iterative and constitutive process is sometimes called the hermeneutic circle.

What has just been said about observables also applies to partitioning a system into subsystems. G. Mahler[21] vigorously pointed out that the identification of subsystems in a complex system may be a highly creative act. In general, subsystems do not preexist in a naïve way but are in a sense created in the constitutive act of their identification. Partitioning may be considered as a special case of constituting observables, because partitioning is achieved by means of partition observables whose different values differentiate between the subsystems. Partition observables and, hence, the associated partitions may be complementary, resulting in an incompatibility of different partitions. Two such incompatible partitions cannot be overlaid in order to arrive at a common refinement of both of them. A simple physical example of such a situation are partitions according to position or momentum of a quantum multiparticle system. In physical systems, the position observable $Q$ is a privileged partition observable, which differentiates between subsystems by their different locations. The paramount importance of the position observable $Q$ is reflected in the fact, that the realm of physics is often identified with the range of applicability of the position observable. This is reminiscent of Descartes' denotion of the material world as "res extensae". Indeed, position and locality play a vital role in physics down to small distances of the order of the Planck length $l_{P}$.

The first partition, prior to and prerequisite for every act of measurement or cognition is the split between observer and observed system. In quantum theory this is referred to as the Heisenberg cut. In the wider framework of GQT we call it the epistemic split. Just as the Heisenberg cut, the epistemic split is movable but not removable, because every

\footnotetext{
${ }^{2}$ In physical quantum theory, as oppposed to GQT, entropy is not an observable in the technical sense.
} 
cognition accessible to us is the cognition of someone about something. The epistemic cut may be far outside the observer, if a remote quasar is observed, or run right through a person's mind in the case of self observation, but it is never absent. It is conceivable that there are partitions and observables incompatible with a given or even any epistemic split. Quantum theoretical uncertainties are closely related to the epistemic split. As already mentioned above, in the quantum theory of physical measurement the time development in the large system containing both observer and observed is completely deterministic. Stochasticity enters into quantum theory as a result of the epistemic split into the subsystems "measured system" and "observer" or "measurement apparatus" and by the subsequent projection onto the latter system and the interpretation of the result obtained there as a statement about the measured system. In quantum physics, there is a symmetry between observer and observed. Projection onto the measured system yields the same probability distributions as the above-mentioned projection onto the measurement device. One may wonder[18], to what extent this symmetry has a generalization to GQT in the form of a certain "inside-outside" symmetry.

Coming back to observables in quantum physics and GQT, it is important to stress, that they already presuppose the epistemic split. Observables neither exclusively pertain to the observed system nor to the observer, rather they are located astride on the epistemic split. Related to this, the observer plays an active role in the constitution of observables, in the choice of the observables to be measured and in the factual establishment of the measurement results.

One might be tempted to associate observables with properties of systems, which, like position or velocity, correspond to substantial entities best expressed by nouns. In [22] we argue, that this would point in the direction of a one-sided preference for an ontology of substances. Process observables associated to transitions and changes of a system and typically better expressible by verbs are of equal importance, and a balance between substance and process ontology [23, 24] seems to be desirable. We shall have to say more about this in the following section.

For what follows we should also keep in mind, that in GQT inner observables occurring in introspective self observation are fully legitimate objects of consideration.

\section{The emergence of physical time}

We already mentioned several times, that, as opposed to internal A-time, external physical B-time lacks an intrinsic directedness as well as the quality of a distinguished "now". The physics of time direction 25] endeavors to establish a physical basis for the evident directedness of time encountered everywhere in the world we live in. In other 
words, a physical mechanism is sought, which endows B-time with directedness. (The equally fundamental problem of the missing "now" is normally not dealt with.) The investigations related to this task proceed in the following way: First various "arrows of time" are described, in which time shows directedness, such as the thermodynamic time arrow for the increase of entropy, the cosmological time arrow for the expansion of the universe, the retardation time arrow for the emission of radiation from a spacially localized source or the evolutionary time arrow for the formation of structures in our world. Then arguments are given, that the various time arrows are aligned, i.e. point into the same direction. Finally, one of the time arrows is identified as primary and a physical mechanism is proposed to account for it. Usually, either the cosmological or the thermodynamical time arrow are placed in the pivotal position. Here, the cosmological time arrow appears in the role of a boundary condition for cosmological evolution, whereas a complete formal deduction of the second law of thermodynamics from the laws of physics is still missing.

In these physical approaches to the directedness of time, the evolutionary time arrow is interpreted as a reflection of the thermodynamic time arrow. This is also offered as the explanation for the directedness of internal A-time, referred to as the "psychological time arrow" in this context.

In this note, we propose to place internal A-time in the primary position as a categorial constitutive feature of the human mode of existence, prior to any physical modelling of the world. Physical B-time would then result secondarily together with a loss of the qualities of directedness and "now". One evident advantage of this view is that it is certainly easier to understand a loss of features than the emergence of completely new features of time. Anyhow, as explained in Section 2, A-time is present in the heart of quantum theory and thermodynamics.

Our scenario for the constitution of physical B-time has been described in detail in [19]. GQT offers itself as a suitable formal framework, because it allows to treat material and mental systems on an equal footing. (For a related but in many respects rather different quantum approach to temporal matter-mind systems see [26].) Here we list the most important steps on this way, referring to [19] for details:

1. After an epistemic split, internal time observables $T_{i}$ arise in subsystems corresponding to individuals and the time bounded mode of their conscious personal existence.

2. The partial synchronization between different internal time observables $T_{i}$ as well as between many "clock" observables $T_{I}$ identified in the outer physical world is certainly not an effect of causal interactions, but an acausal parallelism which can 
be described by entanglement correlations in the sense of GQT between many subsystems.

3. External time is successively sharpened and operationalized in a long and sophisticated process of optimizing physical clock observables such that the afore-mentioned entanglement correlations become as strict as possible. (For a nice account see [27].) At present, the best clocks are given by atomic systems with oscillations between sharp energy levels.

4. In view of what was said in Section 2, time becomes more and more similar to space in this process of externalization. After all, space is the form of the exterior sense. In the course of "spacialization" of time both the notion of "now" and the future directedness of time get lost, because space neither has a privileged "here" nor a natural directedness.

5. We saw that, pushed to the extremes, sharpening of the concept of physical time eventually will lead to its deconstruction with a loss of its fundamental meaning. So, the course of events can be summarized in the following way: establishment of internal time, establishment of physical time along with a fading of characteristic features of inner time, deconstruction of physical B-time.

The distinction between substance and process observables mentioned at the end of Section 5 can be formalized using the time observables $T_{i}[28,22]$. A substance observable $C$ is an observable which commutes with $T_{i}: T_{i} C=C T_{i}$. This means that either $C$ is compatible with a sharp localization in time or bears no reference to time whatsoever. The position observable $Q$ or the angles of an triangle are simple examples of substance observables. Process observables can be defined as complementary to $T_{i}: T_{i} D \neq D T_{i}$. By definition, a temporal process has no precise temporal localization. The complementarity of substance and process observables was proposed in [28, 22] as a resolution of Zeno's paradox: A flying arrow seems to freeze in its motion, when attention is focused on its momentary position at any given instant of time: The arrow never occupies more space than given by its length. The position of the arrow at a given time is a substance observable, whereas its total flight is described by a process observable. The incompatibility of process and substance in this case is similar to the vanishing of the concept of the orbit in the description of motion in quantum mechanics. 


\section{Conditio humana}

Evidently, the world is never given to us directly but only as it appears on our inner screen. This trivial fact, which in philosophical terminology is just the phenomenal character of the world, when taken seriously, has far reaching consequences. Everything we sensually or intellectually conceive of our world is shaped and conditioned in a categorial way by the mode of our existence as conscious individuals. Naive realism asserts that the world appears to us more or less "like it really is". Sometimes our categorial cognitive structure is compared to a pair of colored sunglasses, which can be taken off to allow a look at the real world. But also this optimistic belief underestimates the inexorable phenomenality of our existence, which must be the starting point of every reflection about the way we orient ourselves in our world. In particular, physics cannot lay its own foundations but has to be aware of the categorial prerequisites imposed by our cognitional system and our mode of existence. In this spirit we already mentioned in Section 3 that a measurement should not entirely be conceived as a physical process but also as an act of cognition. This also prevents a complete causal closure of physics. Of course, the physical process accompanying measurement has to be investigated and consistency with the possibility of cognition must be guaranteed. A strict physical reductionism, trying to reduce "everything" to physics, is unaware of the phenomenal character of the world and, hence, of its own foundations. Moreover, as already mentioned, it runs into the naive methodological mistake to identify the model with what is modelled. Everetts's many-world interpretation of quantum theory illustrates the bizarre consequences of an extreme physicalism in the interpretation of measurement: The whole universe, conceived as a purely physical system, is assumed to split into several branches as the result of each measurement.

The main structural features of the phenomenal mode of human existence have already been mentioned in passing. We briefly collect them here, a more detailed analysis can be found in [29]. (Ref. [30] contains a comprehensive and deep discussion.)

- The figure of oppositeness. In every act of cognition we experience ourself as an observer, different and set apart from what we observe. This is sometimes referred to as the egocentricity [31, 32, 33] of human existence. The epistemic cut between observer and observed is never absent.

- Temporality. Human existence is inescapably temporal in the sense of a futuredirected A-time with a privileged "now".

- Factuality. We live in a world of facts rather than a world of potentialities. Everything which appears to us, primarily touches us in the form of a fact. In particular, 
the "now" carries the imprint of prototypic factuality.

These basic existential features are deeply encoded in the structure of quantum theory and GQT [29]. The naturalness and, in a way, a priori structure of quantum theory has been observed by many authors and has, for instance, been expressed in full clarity by M. Bitbol[34].

- The epistemic cut is present in the very special and fundamental role attributed to measurement in quantum theory and GQT. We saw that observables are located right on the epistemic cut. Standard reductive physicalism ignores the importance of the observer and the epistemic cut in favor of the outside world. In this sense, it is as one-sided and implausible as a solipsistic world view, which ignores the outside in favor of the inside world.

- In section 3, we saw, how deeply A-time is encoded into quantum theory, GQT (and thermodynamics).

- Factuality is intimately related to quantum theoretical measurement, which basically amounts to a transition from potentiality to a measurement result of factual validity.

The categorial scheme of human existence is, of course, the product of a long development. The temporality of primitive animals is a total subjection to the undivided factuality of a simple "now". Memory and the possibility of preparing actions open up the horizon of temporality eventually resulting in a differentiation between past, present and future. Causality and personal freedom, which are often considered to be in contradictory relationship, actually rely on one another and are in fact offshoots of the same root of such a developed and differentiated temporality. This phylogenetic process is repeated in quick motion in the ontogenesis of every human individuum. Related to the unfolding of temporality is an emancipation from the tight binding to primitive factuality. Free exploration of the space of possibilities comes into sight with the capacity for hypothetical and contrafactual thinking. Along with this emancipation goes a deepening of the epistemic cut. The precise form of human existence undergoes a process of varied cultural evolution and also shows large individual differences. Development goes on: Man is always rebellious against his categorical limitations. Philosophy, science and arts grant visions on timeless structures. Utopianism challenges factuality and integrative world views embedding man into an comprehensive universe try to alleviate the egocentricity of the epistemic cut. 


\section{References}

[1] J.E. Mc Taggart. The unreality of time. Mind, 17:457-474, 1908.

[2] C. Rovelli. Notes for a breef history of quantum gravity. In 9th Marcel Grossmann Meeting in Roma, July 2000. arxiv:gr-qc/000661v3, updated 2008.

[3] Katrin Becker, Melanie Becker, and John Schwarz. String Theory and M-Theory: A Modern Introduction. Cambridge University Press, 2007.

[4] Th. Thiemann. Introduction to Modern Canonical Quantum General Relativity. Canbridge University Press, 2007.

[5] H. Nicolai, K. Peeters, and M. Zamaklar. Loop quantum gravity: an outside view. Class.Quant.Grav., 22:R193, 2003.

[6] C. Kiefer. Conceptual issues in quantum cosmology. In Kowalski-Glikman, editor, Towards Quantum Gravity, pages 158-187. Springer Publishing Company, 2000.

[7] R. Brunetti and K. Fredenhagen. Time of occurrence observable in quantum mechanics. Phys. Rev. A, 66:044101, 2002.

[8] R. Brunetti, K. Fredenhagen, and M. Hoge. Time in quantum physics: From an external parameter to an intrinsic observable. Found. Phys., 40:1368-1378, 2010.

[9] R. Haag. Local Quantum Physics. Springer-Verlag, 1992.

[10] H. Atmanspacher, H. Römer, and H. Walach. Weak quantum theory: Complementarity and entanglement in physics and beyond. Foundations of Pysics, 32:379-406, 2002.

[11] H. Atmanspacher, T. Filk, and H. Römer. Weak quantum theory: Formal framework and selected applications. In G. Adenier, A. Yu. Khrennikov, and T.M. Nieuwenhuizen, editors, Quantum Theory: Reconsiderations and Foundations, pages 34-46. American Institute of Physics, New York, 2006.

[12] T. Filk and H. Römer. Generalized quantum theory: Overview and latest developments. Axiomathes, 21,2:211-220; DOI 10.1007/s10516-010-9136-6, 2011, http://www.springerlink.com/content/547247hn62jw7645/fulltext.pdf.

[13] H. Atmanspacher. Quantum approaches to consciousness. In E. Zalta, editor, Stanford Encyclopedia of Philosophy, updated 2011. 
[14] H. Atmanspacher and H. Römer. Order effects in sequential measurements of noncommutative psychological observables. Journal of Mathematical Psychology, 56: 274-280, 2012 http://arxiv.org/abs/1201.4685, 2012.

[15] H. Atmanspacher, T. Filk, and H. Römer. Quantum zeno features of bistable perception. Biological Cybernetics, 90:33-40, 2004.

[16] H. Atmanspacher, M. Bach, T. Filk, J. Kornmeier, and H. Römer. Cognitive time scales in a Necker-Zeno model of bistable perception. The Open Cybernetics and Systemic Journal, 2:234-251, 2008.

[17] H. Atmanspacher, T. Filk, and H. Römer. Complementarity in bistable perception. In H. Atmanspacher and H. Primas, editors, Recasting Reality: Wolfgang Pauli's Philosophical Ideas and Contemporary Science. Springer Verlag, 2008.

[18] H. Römer. Verschränkung (2008). In M. Knaup, T. Müller, and P. Spät, editors, Post-Physikalismus, pages 87-121. Verlag Karl Alber, Freiburg i.Br., 2011.

[19] H. Römer. Weak quantum theory and the emergence of time. Mind and Matter, $2: 105-125,2004$.

[20] W. von Lucadou, H. Römer, and H. Walach. Synchronistic phenomena as entanglement correlations in generalized quantum theory. Journal of Consciosness Studies, 14:50-74, 2007.

[21] G. Mahler. The partitioned quantum universe. Mind and Matter, 2:67-91, 2004.

[22] H. Römer. Complementarity of process and substance. Mind and Matter, 4:69-89, 2006.

[23] A.N. Whitehead. Process and Reality. Free Press, London, 1929/1978.

[24] N. Rescher. Process Philosophy: A Survey of Basic Issues. University of Pittsburgh Press, 2000.

[25] D. Zeh. The Physical Basis of the Direction of Time (The Frontiers Collection). Springer, Berlin, Heidelberg, 2009.

[26] H. Primas. Time-entanglement between mind and matter. Mind and Matter, 1:81121, 2003. 
[27] Th. Filk and D. Giulini. Am Anfang war die Ewigkeit. Auf der Suche nach dem Ursprung der Zeit. C. H. Beck, 2004.

[28] H. Römer. Substanz, Veränderung und Komplementarität. Philosophisches Jahrbuch, 113:118-136, 2006.

[29] H. Römer. Why do we see a classical world? Travaux Mathématiques XX: 167-186, 2012 http://arxiv.org/abs/1112.6271

[30] G. Prauss. Die Welt und wir. J. B. Metzeler, Stuttgart, Weimar, 2 vols, 1990, 2006.

[31] E. Tugendhat. Egozentrizität und Mysik. C. H. Beck, München, 2003.

[32] E. Tugendhat and P. Cresto-Dina. Egocentricità e mistica. Bollati Borringheri, 2010.

[33] E. Tugendhat. Egocentricidad y mística: un estúdio antropológico. Editorial Gedisa, 2004.

[34] M. Bitbol. The quantum structure of knowledge. Axiomathes, 21,2:357-371; DOI 10.1007/s10516-10-9129-5, 2011. 